\title{
(C) OPEN ACCESS \\ Anterior but not posterior choroid changed before and during Valsalva manoeuvre in healthy Chinese: a UBM and SS-OCT study
}

\author{
Fei Li, Kai Gao, Xingyi Li, Shida Chen, Wenbin Huang, Xiulan Zhang
}

\begin{abstract}
- Additional material is published online only. To view please visit the journal online (http://dx.doi.org/10.1136/ bjophthalmol-2016-309881).
\end{abstract}

State Key Laboratory of Ophthalmology, Zhongshan Ophthalmic Center, Guangzhou, China

\section{Correspondence to} Professor Xiulan Zhang, Zhongshan Ophthalmic Center, State Key Laboratory of Ophthalmology, Sun Yat-sen University, 54 S. Xianlie Road, Guangzhou 510060, China; zhangx12@mail.sysu.edu.cn

Received 8 November 2016 Revised 8 March 2017 Accepted 26 March 2017 Published Online First 21 April 2017

\section{CrossMark}

To cite: Li F, Gao K, Li X, et al. Br J Ophthalmol 2017;101:1714-1719.

\begin{abstract}
Purpose To determine if the anterior choroid is involved in ocular change during the Valsalva manoeuvre (VM). Materials and methods Fifty-three healthy volunteers aged 18-65 years with normal visual field test results and no history of intraocular pressure (IOP) exceeding $21 \mathrm{~mm} \mathrm{Hg}$ were recruited. Anterior and posterior choroidal changes before and during VM were recorded by ultrasound microscope and swept-source optical coherence tomography, respectively. Parameters of the anterior segment included ciliary body thickness (CBT0), thickness of the choroid at a distance of $4 \mathrm{~mm}$ from the root of the iris (CT4), anterior placement of the ciliary body (APCB) and trabecular-ciliary angle (TCA). Thickness of different layers of retina and posterior choroid were also measured and compared before and during VM. IOP, blood pressure (BP), heart rate (HR), axial length, spherical equivalent refractive error and pupil diameter (PD) were also recorded and analysed.

Results VM caused elevated IOP, systolic BP, diastolic $\mathrm{BP}$ and increased HR. There was a significant increase in anterior parameters including CBTO, CT4 and APCB $(p<0.001)$, but not in TCA or PD ( $p>0.05)$. The mean change of CBTO, CT4 and APCB were: from $1.00 \pm 0.09 \mathrm{~mm}$ to $1.11 \pm 0.10 \mathrm{~mm}(p<0.001)$, from $0.29 \pm 0.04 \mathrm{~mm}$ to $0.36 \pm 0.05 \mathrm{~mm}(p<0.001)$, from $0.76 \pm 0.11 \mathrm{~mm}$ to $0.88 \pm 0.13 \mathrm{~mm}(p<0.001)$, respectively. However, there is no significant change in posterior choroid (from 215.74 $\pm 60.23 \mu \mathrm{m}$ to $214.82 \pm 61.32 \mu \mathrm{m}, \mathrm{p}=0.17$ )

Conclusion We found that VM did not affect the posterior choroid, but it did cause thickening of the anterior choroid and the ciliary body, both of which led to a larger anterior placement of the ciliary body and a narrowed anterior chamber. The anterior (but not the posterior) choroid could be related to IOP elevation and a narrowed anterior chamber in primary angle closure diseases.
\end{abstract}

\section{INTRODUCTION}

Glaucoma is a worldwide ophthalmic disease and, in Asia, a large proportion of the patients suffer from primary angle closure diseases (PACDs). ${ }^{1}$ PACD refers to a spectrum of diseases, including acute primary angle closure (APAC) or chronic primary angle closure (CPAC), primary angle closure glaucoma (PACG) and primary angle closure suspect (PACS). Among them, APAC leads to a rapid increase in intraocular pressure (IOP), causing redness, blurred vision, and pain. The risk factors of APAC vary. A number of factors may trigger an acute attack, such as emotional fluctuations and the intake of several systematically used drugs. ${ }^{2}$ These factors trigger strong autonomic activity leading to a change in ocular blood flow, which changes the thickness of the choroid. ${ }^{3}$

The choroid expansion theory was the first to explain the possible relationship between the choroid and the occurrence of PACD. ${ }^{4}$ The theory described that choroid expansion caused elevated IOP, which leads to increased aqueous feedback, decreased anterior chamber volume and, finally, pupillary block. It was not proven in imaging studies until the invention of the enhanced depth imaging optical coherence tomography (EDI-OCT).

In order to identify the risk factors of PACD, we first measured the anterior and posterior parameters in a healthy Chinese cohort, which demonstrated that female eyes had narrower anterior biometric parameters compared with male eyes. This correlated with increasing age, indicating that age and sex are risk factors of APAC. ${ }^{5}$ We then performed research on different types of PACD. The study by Zhou et $a l^{6}$ found that choroid thickness (CT) in APAC eyes was greater than that of fellow eyes; and a study by Zhou et $\mathrm{al}^{7}$ showed that CT in PACS eyes was greater than that of normal eyes. In another study, which considered CT during and after an acute attack, we found that APAC eyes had a higher level of macular CT than PACS eyes when the IOP was reduced. ${ }^{8}$ These studies regarding PACD patients demonstrated that a thicker posterior choroid was seen in patients with PACD..$^{5-9}$

Whether choroidal change is a cause or result of PACD remains unclear only from the results of cross-sectional studies. Further studies involving the Valsalva manoeuvre (VM) were performed in a different population to discover the exact role that the choroid plays in PACD. VM was first described by the Italian physician, Antonio Maria Valsalva; it is forced exhalation against a closed airway. VM may elicit a series of cardiovascular responses, such as elevated blood pressure (BP), increased thoracic pressure and reduced venous return through a change in autonomic activity. ${ }^{10-12} \mathrm{VM}$ also affects ocular structures and functions and leads to elevated IOP, narrowed anterior chamber width, increased lens vault, and others. ${ }^{11}$ 13-16 Mechanisms underlying these phenomena are not clear, but it is hypothesised that uveal expansion leads to changes in the anterior chamber and the IOP. ${ }^{16}$ Our previous research ${ }^{14}$ proved that only the parameters in the anterior chambers changed during VM 
while the choroid at the posterior pole were not significantly affected, which was consistent with the findings of other studies. However, changes in the anterior choroid have not been clarified during VM.

In order to discover if the anterior choroid is involved in ocular change during VM and the relationship between the changes in different ocular parameters, we performed a prospective study in healthy Chinese subjects. We chose ultrasound microscope (UBM) instead of the anterior segment OCT (AS-OCT) for clearer imaging of the anterior choroid. Measurement of the posterior choroid and other biometric parameters were also recorded. Our aim was to ascertain the reason for changes in the anterior chamber and the possible mechanism of PACD.

\section{METHODS}

\section{Subject recruitment}

All participants involved in the study signed informed consent forms before the examination. We recruited study participants from the Zhongshan Ophthalmic Center of Sun Yat-sen University, Sun Yat-sen University Medical School and nearby communities in Guangzhou, China. The study was approved by the Ethical Review Committee of the Zhongshan Ophthalmic Center and was conducted in accordance with the Declaration of Helsinki for research involving human subjects. We enrolled subjects aged between 18 and 65 years with normal visual field test results and no history of IOP exceeding $21 \mathrm{~mm} \mathrm{Hg}$. Exclusion criteria included: (1) systemic diseases (eg, hypertension, diabetes mellitus and severe cardiopulmonary insufficiency); (2) the use of systemic or topical medications; (3) current ocular diseases (eg, conjunctivitis, cataract and fundus diseases); (4) previous ocular surgery; (5) high myopia or hyperopia (spherical equivalent (SE) refractive error greater than -6 or +3 dioptres); (6) clinically relevant opacities of the optic media and (7) poor compliance in performing VM correctly.

\section{VM training}

We trained participants to do VM before entering the study, as we had done in previous research. ${ }^{14}$ Considering the relatively small space between the study subjects and the instruments, we used a modified way to perform VM, which was developed by Levin. ${ }^{17}$ During VM, each participant first took a deep breath and then blew forcefully against his/her hand and closed glottis while squeezing his/her nose with his/her index finger and thumb. ${ }^{18}$ The examiner counted $15 \mathrm{~s}$ after the participant began to perform VM, then conducted the examinations. VM was sustained during the examinations by maintaining the expiratory pressure against the hand and the glottis. In total, nine VMs were performed during the whole study. Each participant was given a brief rest of $10 \mathrm{~min}$ between two VMs.

\section{Examinations}

Before enrolment, all subjects underwent regular and detailed ocular examinations, including best-corrected visual acuity, slit-lamp examination, fundus examination with a 90-dioptre lens and axial length (AL) measurements by IOL-Master (Carl Zeiss Meditec, La Jolla, California, USA). Systolic BP (SBP) and diastolic BP (DBP) were measured with an electronic sphygmomanometer. IOP was measured by non-contact tonometry (NCT) (CT-80A, Topcon, Tokyo, Japan). We did not choose the Goldmann tonometer to measure IOP because during a contact examination it has the potential to damage the cornea, and NCT can provide a relatively accurate result under normal IOP. A refractive error examination was performed using an

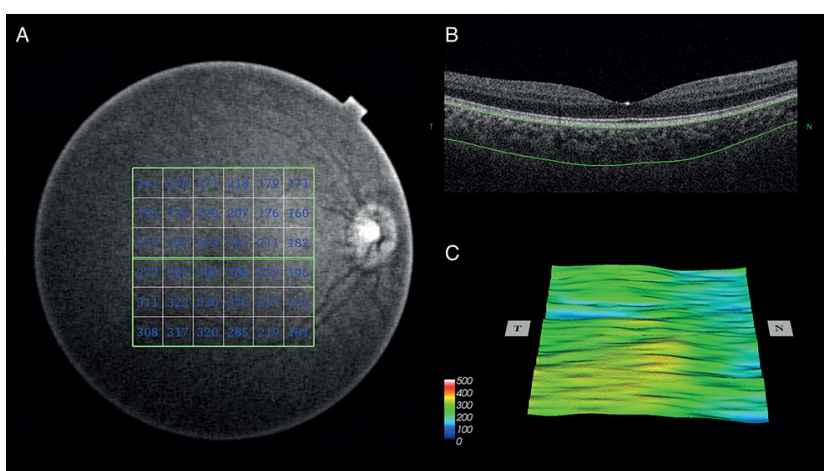

Figure 1 Swept-source optical coherence tomography image showing the automatic measurements of retinal and choroidal thickness. (A) A $6 \times 6 \mathrm{~mm}$ area of the different layers around the macula was automatically created and was divided into $6 \times 6$ grids, and the mean value of the thicknesses of different layers were calculated for the 36 sectors of the grid. (B) Automatic segmentation of the chorioscleral border made by software. (C) Choroidal topographic map of the $6 \times 6 \mathrm{~mm}$ area.

autorefractometer (KR-8900 version 1.07; Topcon, Tokyo, Japan). After baseline information of BP, IOP and refractive error was obtained, VM was performed while repeating the same measurements using the same procedures. Each participant received the examinations in a sitting position. All examinations were performed on the same morning, followed standard operating procedures and were non-contact to avoid the influence of corneal contact on the parameters of the anterior chamber.

\section{Swept-source OCT imaging and measurements}

We obtained images of the posterior choroid in the macular region using the DRI-OCT (Atlantis, DRI OCT-1; Topcon, Tokyo, Japan) as in our previous studies. ${ }^{5} 14$ The DRI-OCT system uses a tunable laser with a centre wavelength of $1050 \mathrm{~nm}$ as a light source, a $100 \mathrm{~nm}$ tuning range and an $8 \mu \mathrm{m}$ axial resolution in tissue. ${ }^{19} \mathrm{~A}$ three-dimensional imaging scan procedure was performed with a $6 \times 6 \mathrm{~mm}$ raster scan centred on the fovea, which was composed of $256 \mathrm{~B}$-scans, each consisting of $256 \mathrm{~A}$-scans (a total of 65536 axial scans/volume), as shown in figure 1. Only the images with a quality score greater than 45 out of 160 were included in the analysis, according to the manufacturer's recommendation. The participants performed VM in a sitting position, and the same scan procedure was repeated before and during VM. All the examinations were performed in the morning at about 10:00 to reduce the influence from diurnal IOP fluctuations. ${ }^{4}$ Images with artefacts, such as motion artefacts, blinking and segmentation failure, were not included in the data analysis. After scanning, only images with a quality of more than 60 were included to ensure accurate measurement. Measurements of the posterior choroidal and retinal layer thicknesses were performed using the swept source OCT (SS-OCT) segmentation software (V.9.12.003.04). These measurements included the thicknesses of the internal limiting membrane (ILM), the ganglion cell layer (GCL) and the ganglion cell complex (GCC). A $6 \times 6 \mathrm{~mm}$ area of the different layers around the macula was automatically created and was divided into $6 \times 6$ grids (figure 1 ), and the mean value of the thicknesses of different layers were calculated for the 36 sectors of the grid. In order to avoid systemic bias, we checked each SS-OCT image for layer segmentation before we accepted the data obtained by automatic measurement using the software. 


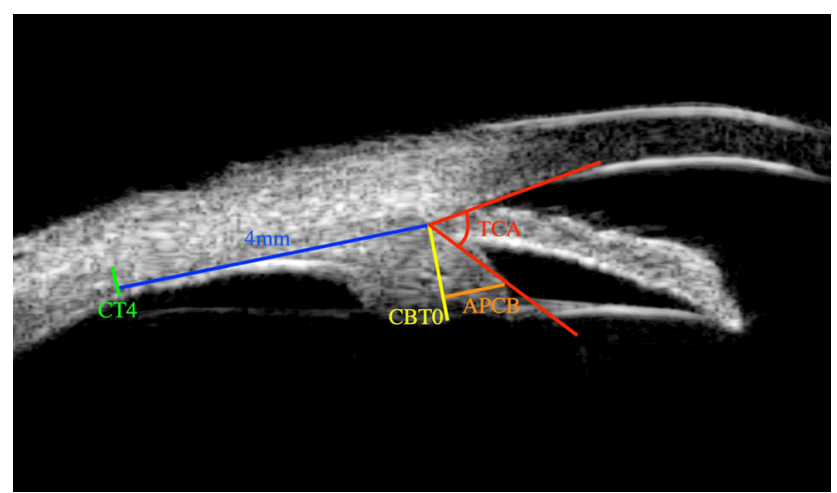

Figure 2 UBM image showing the measurements of $C B T 0, C T 4, A P C B$ and TCA. APCB, anterior placement of the ciliary body; $C B T 0$, ciliary body thickness; CT4, thickness of the choroid at a distance of $4 \mathrm{~mm}$ from the root of the iris; TCA, trabecular-ciliary angle; UBM, ultrasound microscope.

\section{UBM examination and measurements}

Using the Aviso system from Quantel Medicine (Bozeman, Montana, USA), we decided to perform the UBM examination because of its contact with the cornea. The examinations were conducted by a single experienced operator. Participants assumed the supine position during the examination. Images were obtained at the $3,6,9$, and 12 o'clock positions of the eyes while the participant was asked to rotate his/her eyeballs in the opposite direction, thus exposing the anterior choroid. The operator adjusted the contrast and noise during the examination to ensure clear images. The participants then performed $\mathrm{VM}$ in the supine position, and the same scan procedure was repeated. The images were then manually analysed using the Aviso program.

We first determined the location of the root of the iris (the cross point of a line parallel with the inner surface of the cornea and the lower surface of the iris) and then manually measured the parameters below (figure 2): (1) CT4: through the root of the iris, a line is drawn parallel with the inner surface of sclera (CT4 is the thickness of the choroid at a distance of $4 \mathrm{~mm}$ from the root of the iris), (2) CBT0 is the thickness of the ciliary body at the point of the root of the iris; (3) the anterior placement of ciliary body (APCB) is the distance from the most anterior point of the ciliary body to the vertical line from the inner surface of the sclera and (4) trabecular-ciliary angle (TCA) is defined as the angle between the posterior corneal surface and the anterior surface of the ciliary body. ${ }^{20} 21$ All the data were measured independently by two researchers (FL and XL) and Intraclass Correlation Coefficients were calculated to check the consistency of the measurements across both researchers.

\section{Statistical analysis}

The sample size of the study was calculated using PASS V.11. According to data from pre-experiment, in order to have $95 \%$ power to show a $0.04 \mathrm{~mm}$ difference in anterior CT before and during VM with an SD of $0.04 \mathrm{~mm}$ and two-sided type I error rate of 5\%, we needed at least 16 patients. Statistical analyses were performed using Stata V.14.0. The means and SD were calculated for all the measured parameters. Paired t-tests were used to detect the differences in the parameters between baseline and during VM. Univariate and multivariate linear regression was used to determine the relationship between the changes in the anterior chamber parameters. Both univariate and multivariate linear regression was performed using the generalised estimating

\begin{tabular}{|c|c|}
\hline Characteristics & Subjects \\
\hline Number of patients (eyes) & $53(106)$ \\
\hline Mean age, years (SD) & $39.9(15.9)$ \\
\hline Sex (male/female) & $19 / 34$ \\
\hline $\mathrm{AL}, \mathrm{mm}(\mathrm{SD})$ & $23.9(1.3)$ \\
\hline \multicolumn{2}{|l|}{ Parameters } \\
\hline IOP, mm Hg (SD) & $14.76(2.30)$ \\
\hline SE, dioptre (SD) & $-1.59(2.48)$ \\
\hline SBP, mm Hg (SD) & $120.6(12.2)$ \\
\hline DBP, mm Hg (SD) & $73.0(9.6)$ \\
\hline $\mathrm{PD}, \mathrm{mm}(\mathrm{SD})$ & $5.08(0.89)$ \\
\hline \multicolumn{2}{|l|}{ SS-OCT parameters } \\
\hline ILM thickness, $\mu \mathrm{m}$ (SD) & $34.91(4.46)$ \\
\hline GCL thickness, $\mu \mathrm{m}$ (SD) & $72.07(5.43)$ \\
\hline GCC thickness, $\mu \mathrm{m}$ (SD) & $107.00(8.65)$ \\
\hline Retinal thickness, $\mu \mathrm{m}(\mathrm{SD})$ & $272.83(13.49)$ \\
\hline Choroidal thickness, $\mu \mathrm{m}$ (SD) & $215.74(60.23)$ \\
\hline \multicolumn{2}{|l|}{ UBM parameters } \\
\hline CBT0, mm (SD) & $0.99(0.09)$ \\
\hline $\mathrm{CT} 4, \mathrm{~mm}(\mathrm{SD})$ & $0.29(0.04)$ \\
\hline $\mathrm{APCB}, \mathrm{mm}(\mathrm{SD})$ & $0.76(0.12)$ \\
\hline TCA, degree (SD) & $53.6(7.1)$ \\
\hline
\end{tabular}

$A L$, axial length; $A P C B$, anterior placement of the ciliary body; $C B T 0$, ciliary body thickness; $\mathrm{CT} 4$, thickness of the choroid at a distance of $4 \mathrm{~mm}$ from the root of the iris; DBP, diastolic blood pressure; GCC, ganglion cell complex; $\mathrm{GCL}$, ganglion cell layer; ILM, internal limiting membrane; IOP, intraocular pressure; $\mathrm{PD}$, pupil diameter; SBP, systolic blood pressure; $S E$, spherical equivalent; SS-OCT, swept-source optical coherence tomography; TCA, trabecular-ciliary angle.

equations (GEEs) model, with 95\% CIs, taking into account the correlation between the measurements from two eyes. A p value less than 0.10 was considered as statistically significant in univariate linear regression. Variables with a $p$ value of less than 0.10 were taken into multivariate linear regression. A p value of less than 0.05 was considered statistically significant in multivariate regression analysis.

\section{RESULTS}

A total of 53 healthy volunteers (106 eyes) were included in this study. Baseline and demographic characteristics are shown in table 1 . Of those recruited, 34 were women and 19 were men. The mean age of all the participants was $39.91 \pm 15.92$ years. The mean baseline IOP was $14.77 \pm 2.31 \mathrm{~mm} \mathrm{Hg}$. Table 2 shows the changes in baseline and demographic parameters during VM. The mean IOP showed a statistically significant increase from $14.77 \pm 2.31 \mathrm{~mm} \mathrm{Hg}$ to $15.57 \pm 2.92 \mathrm{~mm} \mathrm{Hg}(\mathrm{p}<0.001)$. There was a significant decrease in mean heart rate (HR) from $76.62 \pm 13.40 / \mathrm{min}$ to $72.14 \pm 10.20 / \mathrm{min}$. Mean SBP and DBP showed a significant increase from $120.61 \pm 12.24 \mathrm{~mm} \mathrm{Hg}$ to $124.45 \pm 17.86 \mathrm{~mm} \mathrm{Hg}(\mathrm{p}=0.017)$, and from $72.96 \pm 9.56 \mathrm{~mm} \mathrm{Hg}$ to $80.25 \pm 12.84 \mathrm{~mm} \mathrm{Hg}(\mathrm{p}<0.001)$, respectively. No statistically significant change was found in SE $(\mathrm{p}=0.324)$.

All eyes were included in the analysis of the data from the posterior segment. During VM, no significant change was found in the thickness of the ILM, GCC, GCL, retina or posterior choroid (table 2).

There were 94 eyes of 47 subjects included in the analysis of UBM images of the anterior choroid; images of 12 eyes of six subjects were excluded because of low image quality. Intraclass correlation coefficients revealed good interobserver agreement 
Table 2 Changes in baseline and demographic parameters during VM

\begin{tabular}{|c|c|c|c|c|c|c|}
\hline \multirow[b]{2}{*}{ Parameter } & \multicolumn{2}{|c|}{ During VM } & \multirow[b]{2}{*}{ Mean difference } & \multicolumn{2}{|l|}{$95 \% \mathrm{Cl}$} & \multirow[b]{2}{*}{ p Valuet } \\
\hline & mean & SD & & Lower & Upper & \\
\hline IOP, mm Hg & 15.58 & 2.92 & 0.81 & 0.51 & 1.11 & $<0.001^{*}$ \\
\hline SE, dioptre & -1.61 & 2.49 & -0.02 & -0.07 & 0.02 & 0.32 \\
\hline $\mathrm{SBP}, \mathrm{mm} \mathrm{Hg}$ & 124.4 & 17.9 & 3.84 & 0.3 & 7.4 & $0.02^{*}$ \\
\hline DBP, mm Hg & 80.3 & 12.8 & 7.3 & 4.9 & 9.7 & $<0.001^{*}$ \\
\hline $\mathrm{PD}, \mathrm{mm}$ & 5.10 & 0.92 & 0.02 & -0.07 & 0.11 & 0.65 \\
\hline \multicolumn{7}{|l|}{ SS-OCT parameters } \\
\hline ILM thickness, $\mu \mathrm{m}$ & 34.78 & 4.51 & -0.13 & -0.38 & 0.13 & 0.33 \\
\hline $\mathrm{GCL}$ thickness, $\mu \mathrm{m}$ & 72.24 & 5.66 & 0.17 & -0.25 & 0.6 & 0.42 \\
\hline GCC thickness, $\mu \mathrm{m}$ & 109.52 & 27.91 & 2.54 & -2.76 & 7.85 & 0.34 \\
\hline Retinal thickness, $\mu \mathrm{m}$ & 272.07 & 12.72 & -0.76 & -1.72 & 0.19 & 0.12 \\
\hline Choroidal thickness, $\mu \mathrm{m}$ & 214.82 & 61.32 & -0.92 & -2.24 & 0.4 & 0.17 \\
\hline \multicolumn{7}{|l|}{ UBM parameters } \\
\hline CBT0, mm & 1.10 & 0.10 & 0.11 & 0.101 & 0.119 & $<0.001^{*}$ \\
\hline $\mathrm{CT} 4, \mathrm{~mm}$ & 0.36 & 0.05 & 0.065 & 0.058 & 0.073 & $<0.001 *$ \\
\hline $\mathrm{APCB}, \mathrm{mm}$ & 0.88 & 0.13 & 0.123 & 0.111 & 0.134 & $<0.001^{*}$ \\
\hline TCA, degree & 52.83 & 6.95 & -0.76 & -1.55 & 0.44 & 0.06 \\
\hline
\end{tabular}

*Shows results with a significant difference.

tComparison using paired t-test.

$\mathrm{APCB}$, anterior placement of the ciliary body; CBT0, ciliary body thickness; CT4, thickness of the choroid at a distance of $4 \mathrm{~mm}$ from the root of the iris; DBP, diastolic blood pressure; GCC, ganglion cell complex; GCL, ganglion cell layer; ILM, internal limiting membrane; IOP, intraocular pressure; PD, pupil diameter; SBP, systolic blood pressure; SE, spherical equivalent; SS-OCT, swept-source optical coherence tomography; TCA, trabecular-ciliary angle; UBM, ultrasound microscope; VM, Valsalva manoeuvre.

for measurements of CBT0, CT4, APCB and TCA (supplementary table 1). There was a significant increase in the anterior parameters, including CBT0, CT4 and APCB, but not in TCA or pupil diameter $(\mathrm{PD})$. The mean change of the parameters above was: CBT0 from $1.00 \pm 0.09 \mathrm{~mm}$ to $1.11 \pm 0.10 \mathrm{~mm}(\mathrm{p}<0.001)$, CT4 from $0.29 \pm 0.04 \mathrm{~mm}$ to $0.36 \pm 0.05 \mathrm{~mm}(\mathrm{p}<0.001)$, APCB from $0.76 \pm 0.11 \mathrm{~mm}$ to $0.88 \pm 0.13 \mathrm{~mm} \quad(\mathrm{p}<0.001)$, TCA from $53.60 \pm 7.07^{\circ}$ to $52.83 \pm 6.95^{\circ}(\mathrm{p}=0.062)$ and $P D$ from $5.07 \pm 0.89 \mathrm{~mm}$ to $5.10 \pm 0.92 \mathrm{~mm}(\mathrm{p}=0.651)$. A scatter plot showing change in anterior and posterior choroid was presented in supplementary figure 1 .

The results of univariate and multivariate regression of change in CBT0 ( $\triangle \mathrm{CBT} 0), \mathrm{CT} 4(\triangle \mathrm{CT} 4)$ and APCB $(\triangle \mathrm{APCB})$ are summarised in supplementary table 2 , table 3 and table 4 , respectively. Univariate regression showed that a change in CBT0 was associated with $\triangle \mathrm{IOP}(\mathrm{p}=0.043)$, CBT $(\mathrm{p}=0.017)$, $\triangle$ CT4 $(\mathrm{p}=0.035)$ and $\triangle$ APCB $(\mathrm{p}=0.079)$, but multivariate regression analyses using the GEEs model showed no association between $\triangle$ CBT0 and any other factor. Univariate regression showed that $\triangle \mathrm{CT} 4$ was only associated with SE $(\mathrm{p}=0.08)$ at baseline. $\triangle$ APCB was associated with CT4 $(\mathrm{p}=0.068)$ and change in TCA $(\triangle T C A)(p=0.001)$. Multivariate regression of $\triangle \mathrm{APCB}$ showed $\triangle \mathrm{APCB}$ was associated with $\triangle \mathrm{TCA}(\mathrm{p}=0.001)$.

\section{DISCUSSION}

To our knowledge, this study is the first to evaluate the effects of VM on IOP, BP and both the anterior and posterior choroid, simultaneously. Also, it is the first time that the anteriorbut not the posterior-choroid change during VM has been demonstrated.

We defined a new parameter 'CT4' in order to see a change in 'pure' anterior choroid during VM. To observe the status of the pure choroid during VM, we asked patients to do ocular rotations to expose the anterior choroid at least as far as $4 \mathrm{~mm}$ from the root of the iris when the UBM examination was being performed. At this location, we could obtain data of choroidal change without the influence of the ciliary body.

Our previous studies indicated that a thicker posterior choroid was seen in patients with PACD by EDI-OCT. ${ }^{5-9}$ We then proved that CT has no relationship with disease progression. ${ }^{22}$ However, we cannot tell whether the posterior choroid acted as the reason or the result in PACD from the results of the cross-sectional studies. Studies involving VM provided a solution for this. During VM, increased IOP, a narrowed anterior chamber and a thickened ciliary body were observed using UBM. ${ }^{131523}$ Because VM in our study was modified to accommodate the small space between subjects and examination devices, its effect on physiological changes (including extent of IOP elevation) in our study was weakened. The effect of VM on the posterior segment has been reported in recent years. ${ }^{6} 8924-27$ Schuman et al ${ }^{25}$ studied the effect of VM in wind instrument players and found that IOP fluctuations caused by VM may result in glaucomatous damage. Zhang et $a l^{27}$ detected VM-associated change in the three-dimensional optic nerve head morphology: optic cup-related parameters decreased and neuroretinal rim-related parameters enlarged. Our previous research in healthy subjects demonstrated that VM did not affect CT at the posterior segment. ${ }^{14}$

Clarification is needed to determine whether ocular changes during VM are related to change in the anterior choroid. Previous studies have shown that in patients with APAC, uveal effusion diagnosed by ultrasound biomicroscopy is often seen, which indicates a relationship between APAC and the anterior choroid. $^{28} 29$ Several studies reported data from the anterior segment, which mostly focused on the ciliary body but not on the anterior choroid. ${ }^{13162330}$ Dada et al ${ }^{13}$ studied the influence of VM on the anterior chamber angle in patients with PACG and found significant elevation of IOP, narrowing of the anterior chamber angle recess, thickening of the ciliary body, and an increase in iris thickness. Sihota $e t a l^{23}$ evaluated changes at the anterior chamber angle during $\mathrm{VM}$ in 23 patients with 
primary angle closure and a patent laser peripheral iridotomy. Similar to Dada et $a l^{13}$ they detected an increase in the thickness of the ciliary body and the iris. The angle recess had a positive correlation with the baseline anterior chamber angle $(r=0.41$, $\mathrm{p}=0.05$ ) and a negative correlation with the ciliary body thickness. Wang et $a l^{16}$ carried out research in both angle closure and open angle subjects. The subjects' central anterior chamber depth became shallower, and the anterior chamber angle became narrower. Peripheral iris thickness became thicker, while the central iris thickness did not change, which led to the hypothesis that choroid expansion during VM could be a reason for the narrowed angle. A recent study by Wang et $a l^{30}$ focused on the comparison of parameters in the anterior chamber of APAC eyes with fellow eyes. They found that the ciliary bodies were thinner and more anteriorly rotated in eyes with APAC as well as in their fellow eyes.

In our study, we focused on the change in the anterior choroid and observed thickening of the ciliary body and related changes in the anterior chamber. Our study showed that during VM, IOP increased without a change in the posterior segment but a noticeable change in the anterior segment. Enlargement of the ciliary body and thickening of the anterior choroid narrowed the anterior chamber and pushed the lens up, which led to a shallower anterior chamber. Both CBT0 and CT4 showed an increase. $\triangle \mathrm{CBT} 0$ showed an association with no other factor during VM. Similar to CBT0 and CT4, APCB had a significant increase, and $\triangle \mathrm{APCB}$ showed an association with $\triangle \mathrm{TCA}$. All the results above were concluded after adjusting the influencing factors, such as eye, HR and SBP. Our results on CBT0 change do not agree with those reported by Wang et $a l^{30}$ which could be caused by different baseline characteristics of the study subjects. Although Wang enrolled patients with APAC eyes, the IOP of APAC eyes during their study varied among different patients with a wide range of $7-43 \mathrm{~mm} \mathrm{Hg}$. Thinner CBT0 may be the result of an extremely high IOP, which did not show the real physiological change of the ciliary body during the initiation of an acute attack. Our study showed that $\triangle \mathrm{CBT} 0$ was not correlated with other factors. It indicated that VM directly led to enlargement of ciliary bodies. In short, we hypothesised that a thicker anterior choroid and a smaller APCB seem to be related to a higher chance of developing angle closure diseases. We did not find a significant change in TCA. An explanation for this could be that the enlargement of the ciliary body made itself closer to the centre of the pupil while not causing obvious elevation of the ciliary body. Thus, although the ciliary body was congested and larger, it did not significantly affect the TCA.

The role of the choroid in PACD has been controversial. Arora et $a l^{31}$ found that angle closure eyes had significantly thicker posterior CT than open angle and normal eyes, even after adjusting for the shorter AL, which supports the hypothesis that choroidal expansion contributes to the development of PACD.

Our previous studies, which focused on the posterior CT of different subtypes of PACD, also indicated thicker CT as a risk factor of PACD, which supports Quigley's theory. ${ }^{679}$ Song et $a l^{32}$ demonstrated that during an acute period with elevated IOP, eyes with an acute attack had a thinner choroid compared with fellow eyes with normal IOP. Based on the results from previous studies and our present data, we formulated a new hypothesis: our present data support the role of the anterior choroid as measurable dynamic factor that could contribute to angle closure. Further study is needed to determine if anterior choroidal changes induced by VM can help predict those at higher risk of developing the disease.

\section{Limitations}

One strength of this study is the simultaneous measurements of both the anterior and posterior ocular structures using UBM and SS-OCT, obtaining data of pure anterior choroid during VM. However, the study has some limitations. First, all subjects were enrolled from the same hospital and college, which may have introduced selection bias. Second, data of the posterior segment were measured automatically by software, which may have caused errors, because the machine sometimes gave the wrong segmentation of the different layers. We manually corrected images with the wrong segmentation to avoid incorrect measurements of thickness. Third, we were not able to detect if different durations of VM would have different effects on the choroid, because it was difficult to get the participants to hold their breath for long periods. Finally, anterior and posterior choroids were imaged consecutively, because it is not possible to perform the OCT and the UBM at exactly the same time. And measurements of anterior choroid were obtained in the supine position and in the sitting position for the posterior choroid. Dynamic change of anterior choroid might be affected by higher venous pressure in a supine position.

\section{CONCLUSION}

This is the first study to simultaneously measure both the anterior and posterior choroids for further investigation of the possible mechanism of PACD. We found that VM did not affect the posterior choroid, but it did cause thickening of the ciliary body and the anterior choroid. Thickening of the anterior choroid and the ciliary body led to a larger anterior placement of the ciliary body and narrowed the anterior chamber. IOP elevation and the occurrence of an acute attack could be related to the anterior choroid but not to the posterior choroid.

Contributors All the authors participated in subject enrollment. FL and XZ carried out the study. FL and KG finished data collection and statistical analysis. FL, KG and XZ wrote the paper.

\section{Competing interests None declared.}

Provenance and peer review Not commissioned; externally peer reviewed

Open Access This is an Open Access article distributed in accordance with the Creative Commons Attribution Non Commercial (CC BY-NC 4.0) license, which permits others to distribute, remix, adapt, build upon this work non-commercially, and license their derivative works on different terms, provided the original work is properly cited and the use is non-commercial. See: http://creativecommons.org/ licenses/by-nc/4.0/

(C) Article author(s) (or their employer(s) unless otherwise stated in the text of the article) 2017. All rights reserved. No commercial use is permitted unless otherwise expressly granted.

\section{REFERENCES}

1 Quigley HA. Glaucoma. Lancet 2011;377:1367-77.

2 Razeghinejad MR, Pro MJ, Katz LJ. Non-steroidal drug-induced glaucoma. Eye 2011;25:971-80

3 Zhang X, Wang W, Aung T, et al. Choroidal physiology and primary angle closure disease. Surv Ophthalmol 2015;60:547-56.

4 Quigley HA, Friedman DS, Congdon NG. Possible mechanisms of primary angle-closure and malignant Glaucoma. J Glaucoma 2003;12:167-80.

5 Huang W, Gao X, Li X, et al. Anterior and posterior ocular biometry in healthy Chinese subjects: data based on AS-OCT and SS-OCT. PLOS One 2015;10:e0121740.

6 Zhou M, Wang W, Ding X, et al. Choroidal thickness in fellow eyes of patients with acute primary angle-closure measured by enhanced depth imaging spectral-domain optical coherence tomography. Invest Ophthalmol Vis Sci 2013;54:1971-8.

7 Zhou M, Wang W, Huang W, et al. Is increased choroidal thickness association with primary angle closure? Acta Ophthalmol 2014;92:e514-e520.

8 Wang W, Zhou M, Huang W, et al. Does acute primary angle-closure cause an increased choroidal thickness? Invest Ophthalmol Vis Sci 2013;54:3538-45.

9 Huang W, Wang W, Gao X, et al. Choroidal thickness in the subtypes of angle closure: an EDI-OCT study. Invest Ophthalmo/ Vis Sci 2013;54:7849-53. 
10 Luster EA, Baumgartner N, Adams WC, et al. Effects of hypovolemia and posture on responses to the Valsalva maneuver. Aviat Space Environ Med 1996;67:308-13.

11 Pekel G, Acer S, Yagci R, et al. Impact of Valsalva maneuver on corneal morphology and anterior chamber parameters. Cornea 2014;33:271-3.

12 Porth CJ, Bamrah VS, Tristani FE, et al. The Valsalva maneuver: mechanisms and clinical implications. Heart Lung 1984;13:507-18.

13 Dada T, Gupta V, Deepak KK, et al. Narrowing of the anterior chamber angle during valsalva maneuver: a possible mechanism for angle closure. Eur J Ophthalmol 2006;16:81-91.

14 Li X, Wang W, Chen S, et al. Effects of Valsalva maneuver on anterior chamber parameters and choroidal thickness in healthy chinese: an AS-OCT and SS-OCT study. Invest Ophthalmol Vis Sci 2016;57:189-95.

15 Rafuse PE, Mills DW, Hooper PL, et al. Effects of Valsalva's manoeuvre on intraocular pressure. Can J Ophthalmol 1994;29:73-6.

16 Wang BS, Xiao L, Liu J, et al. Dynamic changes in anterior segment morphology during the Valsalva maneuver assessed with ultrasound Biomicroscopy. Invest Ophthalmol Vis Sci 2012;53:7286-9.

17 Levin AB. A simple test of cardiac function based upon the heart rate changes induced by the Valsalva maneuver. Am J Cardiol 1966:18:90-9.

18 Falcão M, Vieira M, Brito P, et al. Spectral-domain optical coherence tomography of the choroid during valsalva maneuver. Am J Ophthalmol 2012;154:687-92.

19 Mansouri K, Medeiros FA, Tatham AJ, et al. Evaluation of retinal and choroidal thickness by swept-source optical coherence tomography: repeatability and assessment of artifacts. Am J Ophthalmol 2014;157:1022-32.

20 Marchini G, Ghilotti G, Bonadimani M, et al. Effects of $0.005 \%$ latanoprost on ocular anterior structures and ciliary body thickness. J Glaucoma 2003;12:295-300.

21 Wang Z, Huang J, Lin J, et al. Quantitative measurements of the ciliary body in eyes with malignant Glaucoma after trabeculectomy using ultrasound biomicroscopy. Ophthalmology 2014;121:862-9.
22 Li Z, Wang W, Zhou M, et al. Enhanced depth imaging-optical coherence tomography of the choroid in moderate and severe primary angle-closure Glaucoma. Acta Ophthalmol 2015;93:e349-e355.

23 Sihota R, Dada T, Aggarwal A, et al. Does an iridotomy provide protection against narrowing of the anterior chamber angle during Valsalva maneuvre in eyes with primary angle closure. Eye 2008;22:389-93.

24 Cerman E, Eraslan M, Dericioglu V, et al. Choroidal varix elevates macula following Valsalva manoeuvre. Br J Ophthalmol 2014;98:138-40.

25 Schuman JS, Massicotte EC, Connolly S, et al. Increased intraocular pressure and visual field defects in high resistance wind instrument players. Ophthalmology 2000;107:127-33

26 Xie ZG, Yu SQ, Chen X, et al. Macular hole secondary to Valsalva retinopathy after doing push-up exercise. BMC Ophthalmol 2014;14:98.

27 Zhang $Z$, Wang $X$, Jonas JB, et al. Valsalva manoeuver, intra-ocular pressure, cerebrospinal fluid pressure, optic disc topography: Beijing intracranial and intraocular pressure study. Acta Ophthalmol 2014;92:e475-e480.

28 Kumar RS, Quek D, Lee KY, et al. Confirmation of the presence of uveal effusion in Asian eyes with primary angle closure Glaucoma: an ultrasound biomicroscopy study. Arch Ophthalmol 2008;126:1647-51.

29 Sakai $H$, Morine-Shinjyo S, Shinzato $M$, et al. Uveal effusion in primary angle-closure Glaucoma. Ophthalmology 2005;112:413-9.

30 Wang Z, Chung C, Lin J, et al. Quantitative measurements of the ciliary body in eyes with acute Primary-Angle closure. Invest Ophthalmol Vis Sci 2016:57:3299-305

31 Arora KS, Jefferys JL, Maul EA, et al. The choroid is thicker in angle closure than in open angle and control eyes. Invest Ophthalmo/ Vis Sci 2012;53:7813-8.

32 Song W, Huang P, Dong $X$, et al. Choroidal thickness decreased in acute primary angle closure attacks with elevated intraocular pressure. Curr Eye Res 2016;41:526-31. 